\title{
Editorial
}

\section{Bloodstream Infections in Hemodialysis Patients: Getting Some Deserved Attention}

\author{
Jerome I. Tokars, MD, MPH
}

Health care-associated bloodstream infections (BSIs) are a major public health problem. Most surveillance and prevention efforts have been focused on hospital intensive care units. ${ }^{1}$ However, hemodialysis patients have long been known to be at high risk for infection and antimicrobial resistance. Because the incidence of end-stage renal disease doubled in the past decade, the number of hemodialysis patients at risk for BSI and other infections has increased rapidly. At the end of 2000 , approximately 240,000 dialysis patients were treated in 3,700 outpatient facilities in the United States. ${ }^{2}$ This issue of Infection Control and Hospital Epidemiology contains three studies that focus some deserved attention on surveillance and prevention of BSI in hemodialysis patients. ${ }^{3-5}$ A related article discusses catheter-associated BSI in hospital inpatients. ${ }^{6}$

Hemodialysis patients require a vascular access site to withdraw blood so that waste substances can be removed and to replace the blood afterwards. These vascular access sites, listed in order of increasing infection risk, may be native arteriovenous fistulae, synthetic arteriovenous grafts, "permanent" (tunneled, cuffed) catheters, and "temporary" (nontunneled, noncuffed) catheters. Recently, an implanted port device has become available, and it is hoped that this device will have lower associated infection rates than hemodialysis catheters.

Taylor et al. ${ }^{3}$ provide the results of prospective surveillance conducted in 11 Canadian dialysis units for 6 months during 1998 to 1999 . The BSI definition that was used is substantially different from definitions used in the United States. Among other features, this definition would include episodes in which the same organism was isolated from blood and from the intravascular device or skin surface; and single positive cultures for skin contaminant organisms in patients who were immunocompromised (with this term remaining undefined in the article). There were 184 BSIs with an overall BSI rate of 0.59 BSI per 1,000 patient-days (to facilitate comparisons, all BSI rates presented here were converted to BSIs per 1,000 catheterdays; Table). Substantial variation in rates among the 11 centers was noted.

In a second surveillance study, Dopirak et al. ${ }^{5}$ determined rates of BSI among patients at 10 dialysis centers in Connecticut during 1999 to 2000. The Centers for Disease Control and Prevention (CDC) definition for primary BSI was used. A total of 158 BSIs were noted with an overall rate of 0.45 per 1,000 patient-days (Table). Interestingly, rates declined sharply during the course of the study.

Only a few years ago there were few reports of BSI surveillance among hemodialysis patients. In addition to the two studies reported in this issue, two other surveillance studies have recently been reported: one among 6 units in Idaho and Oregon, ${ }^{7}$ and the other among 109 centers participating in a system sponsored by the CDC. ${ }^{8}$ Despite differing definitions and surveillance methods, the reported BSI rates from these 4 surveillance studies are remarkably similar, with 3 of the 4 rounding to $0.6 \mathrm{BSI}$ per 1,000 patient-days overall (Table). This similarity would suggest that adequate results can be obtained with a variety of surveillance methods-the important thing is to do surveillance and use the results to reduce infection rates.

The method used to measure the denominator can make a substantial difference in the amount of work required for BSI surveillance. In studies of hemodialysis patients, BSI rates have been expressed as infections per 1,000 patient-days, 1,000 dialysis sessions ${ }^{3,5,7}$ (patients gen- 
TABLE

BloOdstream Infection Rates Among Hemodialysis Outpatients, By Stud**

\begin{tabular}{|c|c|c|c|c|c|c|c|}
\hline \multirow[b]{2}{*}{ Study } & \multirow[b]{2}{*}{ Type of Study } & \multirow[b]{2}{*}{$\begin{array}{l}\text { No. of } \\
\text { Centers }\end{array}$} & \multicolumn{5}{|c|}{ Vascular Access Type } \\
\hline & & & All & Fistula & Graft & $\begin{array}{l}\text { Tunneled } \\
\text { Catheter }\end{array}$ & $\begin{array}{c}\text { Nontunneled } \\
\text { Catheter } \\
\end{array}$ \\
\hline Taylor et al. ${ }^{3}$ & Surveillance & 11 & 0.59 & 0.09 & 0.23 & 1.31 & 2.23 \\
\hline Dopirak et al. ${ }^{5}$ & Surveillance & 10 & 0.45 & \multicolumn{2}{|c|}{$0.04^{+}$} & \multicolumn{2}{|c|}{$1.14^{\dagger}$} \\
\hline Stevenson et al. ${ }^{7}$ & Surveillance & 6 & 0.65 & 0.07 & 0.15 & 2.37 & 4.53 \\
\hline Tokars et al. ${ }^{8}$ & Surveillance & 109 & 0.59 & 0.08 & 0.18 & 1.61 & 2.91 \\
\hline \multirow[t]{3}{*}{ Price et al. ${ }^{4}$} & Outbreak investigation & 1 & & \multicolumn{4}{|c|}{ Not measured } \\
\hline & Pre-epidemic & & 0.23 & & & & \\
\hline & Epidemic period & & 1.40 & & & & \\
\hline
\end{tabular}

*All rates are bloodstream infections per 1,000 patient-days. For patients with catheters, rates per 1,000 patient-days are equivalent to rates per 1,000 catheter-days. tDetermined for only the last 3 months of the study, after a substantial decrease in the bloodstream infection rate.

erally have 3 hemodialysis sessions per week), 100 patientmonths, ${ }^{4,8}$ or 100 patient-years ${ }^{4}$; all express the same concept, and rates are easily converted from one denominator to another. In the CDC surveillance system, the number of patients at risk is determined only once a month. ${ }^{8}$ If several monthly patient counts are combined, an approximation of patient-time at risk is obtained while reducing the effort required to count individual patient-days or dialysis sessions. In this system, rates are expressed as BSIs per 100 patient-months, a format with a simple and intuitive interpretation: 1.8 BSIs per 100 patient-months (equivalent to 0.6 per 1,000 patient-days) means that each patient has a $1.8 \%$ chance of having a BSI each month.

In a third hemodialysis-related article, Price et al. ${ }^{4}$ report their investigation of an outbreak of BSI at a large outpatient hemodialysis center during 2000 to 2001 . The onset of the outbreak coincided with a change in the facility to for-profit ownership; surveillance for BSI by hospital personnel and the use of prepackaged catheter dressing kits had been discontinued. The BSI rate increased from 0.23 per 1,000 patient-days before the outbreak to 1.4 per 1,000 patient-days during the outbreak (Table). When the outbreak was recognized, staff and patients were re-educated on catheter care, chlorhexidine replaced povidoneiodine for skin antisepsis, and gauze replaced transparent dressings. The BSI rate decreased, with the decrease possibly starting before the interventions. The temporal association of the outbreak with for-profit ownership and discontinuance of surveillance by hospital personnel is significant, considering national trends: during 1985 to 2000 , for-profit ownership increased from $46 \%$ to $78 \%$ and operation independent of hospitals from $56 \%$ to $82 \%$ of outpatient hemodialysis centers. ${ }^{2}$

A fourth article in this issue reports that higher BSI rates in hospitalized patients coincided with a decreased frequency of changing semipermeable transparent catheter dressings (once versus three times per week) and the use of alcohol swabs rather than alcohol sticks during these dressing changes. ${ }^{6}$ Rates declined after these changes were reversed and other interventions were made. It is difficult to draw definite conclusions from this small study, but the authors suggest that changing transparent dressings 3 times a week versus less frequently may lower BSI rates. In contrast, the recently updated $\mathrm{CDC} / \mathrm{Hospital}$ Infection Control Practices Advisory Committee (HICPAC) guideline recommends that dressings over short-term catheters be changed at least every 2 days for gauze dressings and every 7 days for transparent dressings; dressings should also be changed if they become damp, loosened, or soiled, or when inspection of the site is necessary. ${ }^{1}$

The CDC/HICPAC guideline states that either gauze or transparent dressings may be used. ${ }^{1}$ One of the articles in this issue suggests a possible explanation for some reports of higher BSI rates with transparent dressings-interviews revealed that some patients felt the transparent dressings were waterproof and therefore would be protected if they showered. ${ }^{4}$ This highlights that incorrect use, rather than an inherent problem with the transparent dressings, may lead to increased infection rates. Showering was not an explanation for the higher BSI rates with transparent dressings in the other study, however. $^{6}$

Several studies have now reported variation in BSI rates among dialysis facilities. ${ }^{3,5,7,8}$ This variation may be due in part to surveillance artifact (eg, different individuals collecting data or different rates of blood culturing) or to factors not amenable to change (eg, severity of patient illness). However, the variation may also be caused by modifiable factors such as differences in the application of infection control precautions. Further studies may help to explain this variation of rates among dialysis centers and point the way to effective prevention methods.

Articles in this issue ${ }^{3.5}$ and other articles ${ }^{7,8}$ show that hemodialysis catheters have a much higher associated BSI rate than do implanted fistulae or grafts (Table). The single most important strategy to prevent hemodialysis-associat- 
ed BSI is to minimize the use of catheters. Of concern is that an increasing percentage of U.S. hemodialysis patients are being treated with catheters: $13 \%$ during 1995 versus $24 \%$ during $2000 .{ }^{2}$ Efforts to minimize the use of catheters are ongoing and must be pursued vigorously if we are to prevent hemodialysis-related BSI. ${ }^{9}$

As noted above, most BSI surveillance and prevention efforts have been focused on hospital intensive care units, where $80,000 \mathrm{BSIs}$ are estimated to occur yearly. ${ }^{1}$ In comparison, more than 50,000 BSIs would be estimated to occur among hemodialysis outpatients each year using a consensus rate of 0.6 BSI per 1,000 patient-days. Antimicrobial pressure from therapy of these hemodialysisassociated BSIs is one factor driving rates of antimicrobial resistance. Five of the first six U.S. patients with vancomycin intermediate-resistant Staphylococcus aureus infections had received dialysis, ${ }^{10}$ and the first patient found to be infected with a vancomycin-resistant $S$. aureus strain was a hemodialysis patient. ${ }^{11}$ These facts provide powerful motives to expand surveillance and prevention efforts among hemodialysis patients. The problem of infections in hemodialysis patients is an important one that is clearly deserving of our attention.

\section{REFERENCES}

1. Centers for Disease Control and Prevention. Guidelines for the prevention of intravascular catheter-related infections. $M M W R$ 2002;51(RR10):1-29.

2. Tokars JI, Miller ER, Alter MJ, Arduino MJ. National surveillance of dialysis-associated diseases in the United States, 2000. Semin Dial 2002;15:162-171.

3. Taylor G, Gravel D, Johnston L, et al. Prospective surveillance for primary bloodstream infections occurring in Canadian hemodialysis units. Infect Control Hosp Epidemiol 2002;23:716-720.

4. Price CS, Hacek D, Noskin GA, Peterson LR. An outbreak of bloodstream infections in an outpatient hemodialysis center. Infect Control Hosp Epidemiol 2002;23:725-729.

5. Dopirak M, Hill C, Oleksiw M, et. al. Surveillance of hemodialysis-associated primary bloodstream infections: the experience of ten hospitalbased centers. Infect Control Hosp Epidemiol 2002;23:721-724.

6. Curchoe RM, Powers J, El-Daher N. Weekly transparent dressing changes linked to increased bacteremia rates. Infect Control Hosp Epidemiol 2002;23:730-732.

7. Stevenson KB, Hannah EL, Lowder CA, et al. Epidemiology of hemodialysis vascular access infections from longitudinal infection surveillance data: predicting the impact of NKF-DOQI clinical practice guidelines for vascular access. Am J Kidney Dis 2002;39:549-555.

8. Tokars JI, Miller ER, Stein G. A new national surveillance system for hemodialysis-associated infections: initial results. Am I Infect Control 2002;30:288-295.

9. National Kidney Foundation. K/DOQI clinical practice guideline for vascular access, 2000. Am J Kidney Dis 2001;37 (suppl 1):S137-S181.

10. Fridkin SK. Vancomycin-intermediate and -resistant Staphylococcus aureus: what the infectious disease specialist needs to know. Clin Infect Dis 2001;32:108-115.

11. Centers for Disease Control and Prevention. Staphylococcus aureus resistant to vancomycin: United States, 2002. MMWR 2002;51:565-567. 